Pacific Journal of Mathematics

A WILD CANTOR SET IN THE HILBERT CUBE 


\title{
A WILD CANTOR SET IN THE HILBERT CUBE
}

\author{
RAYMOND Y. T. WONG
}

\begin{abstract}
Let $E^{n}$ be the Euclidean $n$-space. A Cantor set $C$ is a set homeomorphic with the Cantor middle-third set. Antoine and Blankinship have shown that there exists a "wild" Cantor set in any $E^{n}$ for $n \geqq 3$, where "wild" means that $E^{n}-C$ is not simply connected. However it is also known that no "wild" Cantor set (in fact, compact set) can exist in many infinite dimensional spaces, such as $s$ (the countably infinite product of lines) or the Hilbert space $l_{2}$. A result of this paper provides a positive answer for a generalization of Blankinship's result in the Hilbert cube.
\end{abstract}

If $X$ is a space, we denote by $X^{n}$ the space $\prod_{i=1}^{n} X_{i}$ and $X^{\infty}$ the space $\Pi_{i=1}^{\infty} X_{i}$ with $X_{i}=X$. Let $\tau_{n}$ denote the projecting function of $X^{\infty}$ onto $X^{n}$ and $\pi_{n}$ the projecting function of $X^{\infty}$ onto $X_{n}$. Let $J, \dot{J}$ denote intervals $[-1,1],(-1,1)$ respectively. The Hilbert cube is the space $J^{\infty}$ under the metric $\rho(x, y)=\sum_{i \geq 1}\left(\left|x_{i}-y_{i}\right|\right) / 2^{i}$. Hilbert space, $l_{2}$, is the space of all square summable sequences of real numbers with metric $d\left(\left(x_{i}\right),\left(y_{i}\right)\right)=\sqrt{ }\left[\sum_{i=1}^{\infty}\left(x_{i}-y_{i}\right)^{2}\right]$. The space $\dot{J}^{\infty}$ is also denoted by $s$. Let $E^{n}=\prod_{i=1}^{n} E_{i}$ be the Euclidean $n$-space.

A Cantor set is a set homeomorphic with the Cantor middle-third set. The existence of a Cantor set $C$ in $E^{n}(n \geqq 3)$ such that $E^{n}-$ $C$ is not simply connected was first demonstrated by Antoine [4] in 1921 and constructed by W. A. Blankinship [5] in 1951. It is known that every Cantor set is $s$ (or in $l_{2}$ ) must be tame, in the sense that its complement in $s$ (or in $l_{2}$ ) is topologically as nics as the space itself. In fact it has been proved (by $\mathrm{V}$. Klee in the case of $l_{2}$ [9] and by R. D. Anderson [1] in the case of $s$, using Klee's method) that if $K$ is a compact set in $X$ (for $X=s$ or $l_{2}$ ), then $X-K \approx X$. The question as to whether a finite dimensional closed set can leave the Hilbert cube multiply connected (in particular, whether a Cantor set can have this property) was then raised in [5] by Blankinship and was also later mentioned in [7] by Klee. In this paper we shall give such a question a positive answer by constructing a Cantor set $C$ in the Hilbert cube $J^{\infty}$ such that $J^{\infty}-C$ is not homotopically trivial. In fact, we shall apply the result of Blankinship [5] to show that $J^{\infty}-C$ has nontrivial 1st-Homotopy group. We remark that such a set $C$ cannot be constructed as a subset of $\dot{J}^{\infty}$. Note that Anderson [1] (by using Klee's method) proved that any Cantor set $C$ (in fact, any compact set) in $\dot{J}^{\infty}$ can be carried into an end-face, say $K_{1}=\left\{x \in J^{\infty} \mid \pi_{1}(x)=1\right\}$, by a homeomorphism on $J^{\infty}$. It is quite clear that the complement of any Cantor subset (in fact, any compact subset) 
of $K_{1}$ in $J^{\infty}$ is homotopically trivial, therefore, if the complement of $C$ in $J^{\infty}$ is to be homotopically nontrivial, $C$ must, in a sense, join various end-faces of $J^{\infty}$.

2. Some notation and lemma. All homeomorphisms concerned are assumed to be geometric homeomorphisms, and when a homeomorphism has domain in $E^{n}$, it is assumed to be linear. Two subsets of $E^{n}$ are similar if they are homeomorphic under some homeomorphism. Let $\Delta$ denote the boundary of the unit square in $E^{2} . A^{*}$-circle is a set homeomorphic to $\Delta$. An $n$-tube, $n \geqq 3$, is a set homeomorphic to the product of a circular 2-cell with $(n-2){ }^{*}$-circles.

We shall choose a fixed set of positive real numbers $r_{1}, r_{2}, \ldots$ with the properties that (1) $r_{1}>1$ and (2) $r_{n+1}>2\left(\sum_{i=1}^{n} r_{i}\right)$. Let $L_{i}=$ $\left[r_{i}=1, r_{i}+1\right] \subset E_{i}$ and $L^{n}=\prod_{i=1}^{n} L_{i} \times\left(r_{n+1}, r_{n+2}, \cdots\right)$. We shall regard $E^{n}$ as a subset of $E^{n+1}$ by considering $E^{n}$ as $E x^{n} 0$.

Lemma 1. If $X$ is a Hausdorff space and $A_{1}, A_{2}, \cdots$ is a decreasing sequence of compact subsets of $X$ such that each $A_{i}$ is dense in itself, then $\mathrm{U}_{i=1}^{\infty} A_{i}$ is dense in itself.

Proof. If $x$ is an isolated point of $\bigcap_{i=1}^{\infty} A_{i}$, then for some $i, x$ is an isolated point of $A_{i}$, contrary to the hypothesis.

3. Brief outline of the construction. The construction is an inductive modification of the construction by Antoine [4] and by Blankinship [5]. The Cantor set $C$ will be the intersection of a decreasing sequence of compact subsets $K_{1}, K_{2}, \cdots$ of the Hilbert cube $L^{\infty}=\prod_{i=1}^{\infty} L_{i}$. For each $n \geqq 3, K_{n}$ will be the product of a compact subset $K_{n}^{\prime}$ of $L^{n}$ with $\prod_{i=n+1}^{\infty} L_{i}$. $\quad K_{3}^{\prime}$ is the intersection of a simple chain of linking 3 -tubes of $E^{3}$ with $L^{3}$. $K_{4}^{\prime}$ will be contained in $K_{3}^{\prime} \times$ $L_{4}$ and is the intersection of a simple chain of linking 4-tubes of $E^{4}$ with $L^{4}$ and so on.

\section{Construction of $K_{3}$.}

DeFinition. Let $r, s$ be positive integers and $d_{r}$ an arbitrary real number. Let $S$ be a compact subset of $E^{\infty}\left(=\prod_{i=1}^{\infty} E_{i}\right)$ such that $\pi_{r}(S)=d_{r}$. We say $\widetilde{S}$ is the set generated by rotating $S$ about the hyperplane $x_{r}=d_{r}$ and $x_{s}=0$ if

$$
\widetilde{S}=\left\{\begin{array}{c}
x \in E^{\infty}: \exists y \in S \ni\left(x_{r}, x_{s}\right) \in \operatorname{Bd}\left(\left[d_{r}-y_{s}, d_{r}+y_{s}\right] \times\left[-y_{s}, y_{s}\right]\right) \\
\text { and } x_{i}=y_{i} \text { for } i \neq r, s
\end{array}\right\}
$$

where $\left[d_{r}-y_{s}, d_{r}+y_{s}\right] \subset E_{r},\left[-y_{s}, y_{s}\right] \subset E_{s}$. 
The following Lemma is evident:

Lemma 2. Suppose $S$ is the set defined above and $\pi_{s}(S)>0$, then $\widetilde{S}$ is homeomorphic to the product of $S$ with $a^{*}$-circle.

DEFINITION. Let

$$
\begin{aligned}
& T^{2}=\left\{x \in E^{\infty}:\left(x_{1}-r_{1}\right)^{2}+\left(x_{2}-r_{2}\right)^{2} \leqq\left(\frac{1}{4}\right)^{2} \text { and } x_{i}=r_{i} \text { for } i \geqq 3\right\} \\
& \Delta_{0}=\left\{x \in E^{\infty}:\left(x_{1}-r_{1}\right)^{2}+\left(x_{2}-r_{2}\right)^{2}=\left(\frac{1}{2}\right)^{2} \text { and } x_{i}=r_{i} \text { for } i \geqq 3\right\} .
\end{aligned}
$$

For $n \geqq 3$, define $T^{n}$ inductively to be the set generated by rotating $T^{n-1}$ about the hyperplane $x_{n-1}=0, x_{n}=r_{n}$.

LEMMA 3 . For $n \geqq 2$, $\min \pi_{n}\left(T^{n}\right) \geqq 1$.

Proof. It is clear for $n=2$. For $n \geqq 3$, it follows from the fact $\min \pi_{n}\left(T^{n}\right)=r_{n}-\left(1 / 4+r_{2}+\cdots+r_{n-1}\right)$ and from the hypothesis of $r_{i}$.

Lemma 4. For $n \geqq 3, T^{n}$ is an $n$-tube in $E^{n}$.

Proof. $\pi_{2}\left(T^{2}\right)>0$ by Lemma 3 . Then by Lemma $2, T^{3}$ is a $3-$ tube. Inductively, $T^{n}$ is an $n$-tube.

Lemma 5. For $n \geqq 3, T^{n} \cap L^{n}=\tau_{2}\left(T^{2}\right) \times \prod_{i=3}^{n} L_{i} \times\left(r_{n+1}, r_{n+2}, \cdots\right)$.

Proof. This is a consequence of Lemma 3.

Let $\left\{t_{t}^{3}\right\}_{i=1}^{l}$ be a chain of cylically linked disjoint 3-tubes contained in the interior of $T^{3}$ and looping once around the axis of $T^{3}$. We assume (1) they are all similar to $T^{3}$, (2) $l \equiv 0(\bmod 4)$ and $l$ is large enough so that each $t_{t}^{3}$ can be regarded as the set generated by rotating a small circular 2-cell $t_{i}^{2}$ along a small *-circle $\Delta_{i},(3) \operatorname{diam}\left(t_{i}^{3}\right)<$ $1 / 3\left(\operatorname{diam} T^{3}\right)$ for all $i$, and (4) Only two members of $\left\{t_{i}^{3}\right\}_{i=1}^{l}$ intersect $\mathrm{Bd}\left(L^{3}\right)$ (one in each side) and the intersection of each such $t_{i}^{3}$ with $\mathrm{Bd}$ $\left(K^{3}\right)$ is exactly two disjoint 2-cells. Let $A_{3}=\bigcup_{i=1}^{k} t_{i}^{3}, K_{3}^{\prime}=A_{3} \cap L^{3}$ and $K_{3}=K_{3}^{\prime} \times \prod_{i=4}^{\infty} L_{i}$.

5. Construction of $K_{4}, K_{5}, \cdots$. For the purpose of simplicity, we shall give only the construction of $K_{4}$ and assert that for $n \geqq 5$, $K_{n}$ can be inductively constructed.

Step 1. For each $i$, let $h_{i}$ be a (linear) homeomorphism of $T^{3}$ 
onto $t_{i}^{3}$. Hence $\left\{t_{i j}^{3}=h_{i}\left(t_{j}^{3}\right)\right\}_{j=1}^{l}$ is a similar chain of cyclically linked disjoint 3 -tubes in $t_{i}^{3}$. We require that each $h_{i}$ is so chosen that (1) if $t_{i}^{3}$ is a member that intersects $\operatorname{Bd}\left(L^{3}\right)$, then only two members of $\left\{t_{i j}^{3}\right\}_{j=1}^{l}$ intersect $\operatorname{Bd}\left(L^{3}\right)$ and the intersection of each such member with $\operatorname{Bd}\left(L^{3}\right)$ is exactly two disjoint 2-cells and $(2) \operatorname{diam}\left(t_{i j}^{3}\right)<\left(1 / 3^{2}\right) \operatorname{diam}\left(T^{3}\right)$ for all $i j$.

Step 2. For each $i, j$, let $t_{i j}^{4}$ be the 4 -tube in $T^{4}$ generated by rotating $t_{i j}^{3}$ about planes $x_{3}=0, x_{4}=r_{4}$. We now regard each $t_{i j}^{3}$ as the set generated by rotating a small 2-cell $t_{i j}^{2}$ along a small *-circle. We assume further that $t_{i j}^{2}$ is contained in $L^{3}$ whenever $t_{i j}^{3}$ intersects $L^{3}$. Let $\tilde{t}_{i j}^{2}$ be the set generated by rotating $t_{i j}^{2}$ about planes $x_{2}=0$, $x_{4}=r_{4}$. Then $t_{i j}^{4}$ can be regarded as the geometric product of $\tilde{t}_{i j}^{2}$ with $\Delta_{i j} . \widetilde{t}_{i j}^{2}$ is a 3-tube. Let $h_{i j}$ be a linear homeomorphism of $T^{3}$ onto $\widetilde{t}_{i j}^{2}$. Let $t_{i j k}^{3}=h_{i j}\left(t_{k}\right), k=1,2, \cdots, l$. We require each $h_{i j}$ is so chosen that (1) if $t_{i j}^{2} \subset L^{3}$, then only two members of $\left\{t_{i j k}^{3}\right)_{k=1}^{l}$ intersect $L^{3} \times \operatorname{Bd}\left(L_{4}\right)$ (one in each side) and the intersection of each such member with $L^{3} \times \operatorname{Bd}\left(L_{4}\right)$ is exactly two disjoint 2-cells and (2) diam $\left(t_{i j k}\right)<(1 / 3)\left(\operatorname{diam} T^{3}\right)$. Let $t_{i j k}^{4}$ denote the geometric product of $t_{i j k}^{3}$ with $\Delta_{i j}$. Let $A_{4}=\bigcup_{i, j, k=1}^{l} t_{i j k}^{4}, K_{4}^{\prime}=A_{4} \cap L^{4}$ and $K_{4}=K_{4}^{\prime} \times \prod_{i=5}^{\infty} L_{i}$.

\section{Theorem 1. Let $C=\bigcap_{i=3}^{\infty} K_{i}$. Then $C$ is a Cantor set in $L^{\infty}$.}

Proof. It follows from the construction that $K_{3}, K_{4}, \cdots$ is a decreasing sequence of compact subset of $L^{\infty}$ and each $K_{i}$ is dense in itself. Hence $C$ is dense in itself by Lemma 1. Furthermore, each $K_{i}$ is a finite union of disjoint compact subsets whose diameters are uniformly small and tend to zero as $i \rightarrow \infty$. We conclude then that $C$ is a compact zero-dimensional space which is dense in itself, hence is a Cantor set.

THEorem 2. If $F$ is a mapping of $\Delta_{0} \times I$ into $L^{n}(n \geqq 3)$ such that $\left.F\right|_{\Delta_{0} \times 0}=$ identity on $\Delta_{0}$ and $F\left(\Delta_{0} \times 1\right)$ is a point, then $F\left(\Delta_{0} \times I\right) \cap$ $K_{n}^{\prime} \neq \phi$.

Proof. The proof is due to [5]. Basically Blankinship had constructed a Cantor set $C^{\prime}$ in $A_{n}$ such that $C^{\prime}$ links $\Delta_{0}$ in $E^{n}$, hence $A_{n}$ also links $\Delta_{0}$ in $E^{n}$. As a consequence, $K_{n}^{\prime}=A_{n} \cap L^{n}$ links $\Delta_{0}$ in $L^{n}$.

THEorem 3. $L^{\infty}-C$ has nontrivial lst-Homotopy group.

Proof. Let $F$ be a mapping of $\Delta_{0} \times I$ into $L^{\infty}$ such that $\left.F\right|_{\Delta_{0} \times 0}=$ 
identity on $\Delta_{0}$ and $F\left(\Delta_{0} \times 1\right)$ is a point. For each $n \geqq 3, \tau_{n}\left(F^{\prime}\right)$ is a mapping of $\Delta_{0} \times I$ into $L^{n}$ satisfying $\left(\tau_{n} F\right)_{\Delta_{0} \times 0}=$ identity on $\Delta_{0}$ and $\left(\tau_{n} F\right)\left(\Delta_{0} \times 1\right)$ is a point. Hence by Theorem $2,\left(\tau_{n} F\right)\left(\Delta_{0} \times I\right) \cap K_{n}^{\prime} \neq$ $\phi$. This implies $F\left(\Delta_{0} \times I\right) \cap K_{n} \neq \phi$, hence $F\left(\Delta_{0} \times I\right) \cap C \neq \phi$.

THeOREM 4. There exist two Cantor sets in the Hilbert cube such that no homeomorphism of one onto the other can be extended to a homeomorphism on the whole Hilbert cube.

$$
\text { Let } \dot{L}_{i}=\operatorname{Int}\left(L_{i}\right) \text { and let }(\dot{L})^{\infty}=\prod_{i=1}^{\infty} \dot{L}_{i} \text {. Let } V_{n}^{\prime}=K_{n}^{\prime} \cap \operatorname{Int}\left(L^{n}\right)
$$
and $V_{n}=V_{n}^{\prime} \times \prod_{i=n+1}^{\infty} \dot{L}_{i}$. Then each $V_{n}$ is a closed subset of $(\dot{L})^{\infty}$ and hence $C_{0}=\bigcap_{n=3}^{\infty} V_{n}$ is both zero-dimensional and closed in $(\dot{L})^{\infty}$. By similar reasoning $C_{0}$ links $\Delta_{0}$ in $(\dot{L})^{\infty}$. Finally, using the fact $s \simeq$ $(\dot{L})^{\infty}$ and $l_{2} \cong s[2]$, we conclude:

THEOREM 5. $s$ and $l_{2}$ contain zero-dimensional closed sets whose complements are not simply-connected.

\section{REFERENCES}

1. R. D. Anderson, Topological properties of the Hilbert cube and the Infinite product of open intervals Trans. Amer. Math. Soc. 126 (1967), 200-216.

2. - Hilbert space is homeomorphic to the countable infinite product of lines, Bull. Amer. Math. Soc. 72 (1966), 515-519.

3. - (Abstract), On Extending Homeomorphisms on the Hilbert cube, Notice Amer. Math. Soc., Vol. 13, No. 3, April 1966, p. 375.

4. L. Antoine, Sur l'homeomorphie de deux figures et de leurs voisinages, J. Math. Pures, Appl. 86 (1921), 221-235.

5. W. A. Blankship, Generalization of a construction of Antoine, Ann. of Math. $\mathbf{5 3}$ (1951), 276-297.

6. V. Klee, Convex bodies and periodic homeomorphisms in Hilbert space, Trans. Amer. Math. Soc. 74 (1953), 36.

7. - Homogeneity of infinite-dimenssonal parallotopes, Ann. of Math. (2) 66 (1957), 454-460.

8. - Some topological properties of convex sets, Trans. Amer. Math. Soc. 78 (1955), 30-45.

9. - A note on topological properties of normed linear spaces, Proc. Amer. Math. Soc. 7 (1956), 673-674.

Received October 28, 1966, and in revised form May 1, 1967. This paper is a part of the author's doctoral thesis under the direction of Professor R. D. Anderson and revised into its present form while the author held a National Science Foundation Grant GP-5860, at UCLA 1966-1967.

Louisiana State University, Baton Rouge

UNIVERSITY OF CALIFORNIA, LOS ANGELES 



\section{PACIFIC JOURNAL OF MATHEMATICS}

\section{EDITORS}

\section{H. ROYDEN}

Stanford University

Stanford, California

\section{J. P. Jans}

University of Washington

Seattle, Washington 98105

\section{J. DUGUNDJI}

Department of Mathematics

Rice University

Houston, Texas 77001

RICHARD ARENS

University of California

Los Angeles, California 90024

\section{ASSOCIATE EDITORS}
E. F. BeCKenbaCH
B. H. NeumanN
F. WOLF
K. YosIDA

\section{SUPPORTING INSTITUTIONS}

UNIVERSITY OF BRITISH COLUMBIA CALIFORNIA INSTITUTE OF TECHNOLOGY UNIVERSITY OF CALIFORNIA MONTANA STATE UNIVERSITY UNIVERSITY OF NEVADA NEW MEXICO STATE UNIVERSITY OREGON STATE UNIVERSITY UNIVERSITY OF OREGON OSAKA UNIVERSITY UNIVERSITY OF SOUTHERN CALIFORNIA
STANFORD UNIVERSITY UNIVERSITY OF TOKYO UNIVERSITY OF UTAH WASHINGTON STATE UNIVERSITY UNIVERSITY OF WASHINGTON

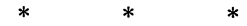

AMERICAN MATHEMATICAL SOCIETY CHEVRON RESEARCH CORPORATION TRW SYSTEMS

NAVAL ORDNANCE TEST STATION 


\section{Pacific Journal of Mathematics}

\section{Vol. 24, No. $1 \quad$ May, 1968}

Harry P. Allen, Lie algebras of type $D_{4}$ over algebraic number fields ...... 1

Charles Ballantine, Products of positive definite matrices. II............ 7

David W. Boyd, The spectral radius of averaging operators ............ 19

William Howard Caldwell, Hypercyclic rings ................... 29

Francis William Carroll, Some properties of sequences, with an application

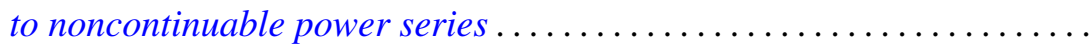

David Fleming Dawson, Matrix summability over certain classes of

sequences ordered with respect to rate of convergence ........... 51

D. W. Dubois, Second note on David Harrison's theory of preprimes. . . . . 57

Edgar Earle Enochs, A note on quasi-Frobenius rings.............. 69

Ronald J. Ensey, Isomorphism invariants for Abelian groups modulo bounded groups ................................ 71

Ronald Owen Fulp, Generalized semigroup kernels ................ 93

Bernard Robert Kripke and Richard Bruce Holmes, Interposition and approximation ................................. 103

Jack W. Macki and James Sai-Wing Wong, Oscillation of solutions to second-order nonlinear differential equations ..................

Lothrop Mittenthal, Operator valued analytic functions and generalizations

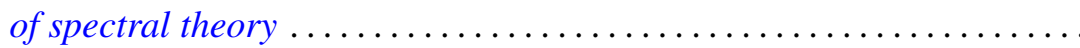

T. S. Motzkin and J. L. Walsh, A persistent local maximum of the pth power deviation on an interval, $p<1 \ldots \ldots \ldots \ldots \ldots \ldots \ldots \ldots \ldots \ldots . \ldots \ldots$

Jerome L. Paul, Sequences of homeomorphisms which converge to homeomorphisms ...........................

Maxwell Alexander Rosenlicht, Liouville's theorem on functions with elementary integrals.

Joseph Goeffrey Rosenstein, Initial segments of degrees .

$\mathrm{H}$. Subramanian, Ideal neighbourhoods in a ring ............

Dalton Tarwater, Galois cohomology of abelian groups . .

James Patrick Williams, Schwarz norms for operators ... .

Raymond Y. T. Wong, A wild Cantor set in the Hilbert cube. 